JOURNAL of

MAINE MEDICAL CENTER Journal of Maine Medical Center

\title{
Missed Opportunities: Documentation and Referral Rates Among Children and Adults with Obesity
}

Heidi Walls

Maine Medical Center

Et al.

Follow this and additional works at: https://knowledgeconnection.mainehealth.org/jmmc

Part of the Primary Care Commons

\section{Recommended Citation}

Walls, Heidi; Holt, Christina; Haskins, Amy PhD; and Dexter, Williams (2021) "Missed Opportunities: Documentation and Referral Rates Among Children and Adults with Obesity," Journal of Maine Medical Center. Vol. 3 : Iss. 2 , Article 4.

Available at: https://knowledgeconnection.mainehealth.org/jmmc/vol3/iss2/4 https://doi.org/10.46804/ 2641-2225.1082

The views and thoughts expressed in this manuscript belong solely to the author[s] and do not reflect the opinions of the Journal of Maine Medical Center or MaineHealth.

This Original Research is brought to you for free and open access by Maine Medical Center Department of Medical Education. It has been accepted for inclusion in the Journal of Maine Medical Center by an authorized editor of the MaineHealth Knowledge Connection. For more information, please contact Dina McKelvy mckeld1@mmc.org.

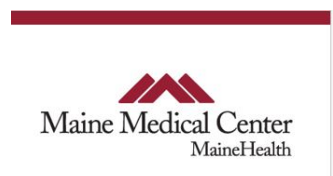


Missed Opportunities: Documentation and Referral Rates Among Children and Adults with Obesity

Authors

Heidi Walls, Christina Holt, Amy Haskins PhD, and Williams Dexter 


\title{
Missed Opportunities: Documentation and Referral Rates among Children and Adults with Obesity
}

\author{
Heidi Walls, MD, ${ }^{1}$ Christina Holt, MD, MSc, ${ }^{2}$ Amy Haskins, $\mathrm{PhD},{ }^{2}$ William Dexter, $M D^{1}$ \\ ${ }^{1}$ Maine Medical Center, Department of Sports Medicine, Portland ME, ${ }^{2}$ Maine Medical Center, Department of Family \\ Medicine, Portland, ME
}

$\begin{array}{ll}\text { Introduction: } & \text { Our study reports rates of obesity documentation on the problem list }(\mathrm{PL}) \text { and numbers of referrals to } \\ \text { obesity medicine specialists and dietitians among pediatric and adult patients with obesity. }\end{array}$

Methods:

All pediatric and adult patients with obesity seen at 14 primary care clinics between 7/1/2017 and $6 / 30 / 2019$ were evaluated for inclusion of obesity on the PL and referrals to obesity medicine specialists or dietitians.

Results: $\quad$ For children with $\mathrm{BMI} \geq 95 \%$ for age, obesity was documented in $31.2 \%$, and $12.5 \%$ received a referral. For adults with $\mathrm{BMI} \geq 30$, obesity was documented in $54.2 \%$, and $8.4 \%$ received a referral. Significantly more subjects received referrals when obesity was on the $\mathrm{PL}$ (both age-groups) compared to those without (children: $20.2 \%$ vs $9.0 \%$; adults: $12.12 \%$ vs $3.9 \%$, p values < 0.0001 ). Higher $\mathrm{BMI}$ and more comorbidities were also associated with higher referral rates (children: $26.6 \%$ vs $8.6 \%$ for those with a BMI $\geq 99$ percentile; adults: $19.9 \%$ vs $5.8 \%$ for those with a BMI $\geq 40$; children: $20.2 \%$ vs $10.7 \%$ for $\geq 1$ comorbidity vs 1 or fewer; adults: $22.7 \%$ vs $5.1 \%$ with $\geq 3$ comorbidities compared to 0 comorbidities).

Discussion: The low rates of documentation of obesity and low rates of referral raise concern that providers may be missing opportunities to identify and manage their patients affected by obesity.

Conclusions: Children and adults with obesity are more likely to be referred to a dietitian or obesity medicine specialist if obesity is on the PL, they have a higher BMI, and they have more medical comorbidities.

Keywords: $\quad$ obesity, documentation, referrals, interdisciplinary care

$\mathrm{O}$ besity is a condition caused by a complex interplay among genetics, the environment, and many other factors. It has become a serious public health concern that currently affects over $1 / 3$ of the population in the United States. The prevalence of obesity in the United States increased from $30.5 \%$ in $1999-2000$ to $42.4 \%$ in $2017-2018$ (1). State-level obesity rates vary considerably, but Maine ranks $28^{\text {th }}$ among all 50 states for highest rates of obesity, and has the highest rate compared to other New England states (2). Obesity is associated with an increased risk of morbidity and all-cause mortality. The biomedical, psychosocial,

Correspondence: Heidi Walls, MD

Maine Medical Center

Department of Sports Medicine

119 Gannet Drive

South Portland, ME 04106

hlwalls@gmail.com and economic consequences of obesity have significant public health implications.

Evidence based treatment for individuals with obesity includes behavioral and nutritional interventions, medical treatment, and even surgery. Primary care providers are well positioned in the US healthcare system to identify patients with obesity and connect them with evidence-based treatments. Unfortunately, the 15-minute office visit model leaves little time for counseling in regards to nutrition and physical activity, and there is strong evidence to suggest that many primary care providers may not have enough expertise in nutrition and exercise to confidently counsel their patients (3). Combating the obesity epidemic will require a multidisciplinary team-based approach and demonstrating to insurance companies the value in reimbursing for obesity related care that 
includes not only bariatric surgery and weight loss medications, but also exercise prescriptions and dietary guidance.

Despite the time constraints placed on primary care providers, there are actions that have been shown to systematically improve obesity related care. Studies have shown that simply documenting obesity on the problem list promotes action about obesity (4). Providers who document obesity are more likely to address it at subsequent visits (4). The USPSTF recommends screening all children (6 years and older) and adults for obesity and offering or referring those with obesity to intensive, multicomponent behavioral interventions $(5,6)$. The purpose of this study was to determine the rate of documentation of obesity on the problem list and referrals to obesity medicine specialists and dietitians among pediatric and adult patients with obesity at 14 primary care offices within the Maine Medical Partners healthcare network. Obesity was defined as $\mathrm{BMI} \geq 95 \%$ ile for age (pediatrics) and $\mathrm{BMI} \geq 30$ (adults).

\section{METHODS}

We performed a retrospective data review including all patients with obesity between 5 - 100 years old, who were seen at any of the 14 primary care clinics within our healthcare system located in Maine between July 1, 2017 and June 30, 2019. De-identified data was obtained from Epic (the electronic health record [EHR]) for each of these locations, which included Portland (population about 66,000) and the surrounding suburban communities: Family Medicine in Portland, Falmouth, Westbrook, Scarborough; Internal Medicine in Falmouth, Westbrook, Scarborough, Cape Elizabeth; Pediatrics in Portland, Falmouth, Westbrook, South Portland, Saco; Lakes Region Primary Care in Windham.

Variables included age, sex, BMI, inclusion of obesity on the problem list, associated ICD-10 diagnosis codes for obesity, metabolic syndrome, sedentary lifestyle, physical deconditioning, diabetes, hypertension, sleep apnea, polycystic ovarian syndrome, acanthosis nigricans, hyperglycemia, dietary counseling, exercise counseling), and any referrals to a dietitian or Obesity Medicine Specialty Clinic within the 2-year study period. The pediatric Obesity Medicine Specialty Clinic in our healthcare system is "Countdown to a Healthy
Me" and provides comprehensive multidisciplinary care with pediatricians, dietitians, and LCSWs. The adult Obesity Medicine Specialty Clinic in our healthcare system is "Weight and Wellness Center" and provides comprehensive multidisciplinary care with physicians, bariatric surgeons, dietitians, and LCSWs. Referrals were treated as a binary variable, yes or no, describing whether they had ever received a referral within the 2-year study period. The percentage of patients whose problem list included obesity and percent of patients with specialty care referrals were calculated for both pediatric and adult patients.

We further categorized BMI as $95-98 \%$ ile vs. $\geq 99$ percentile for children and 30-34.9, 35-39.9, and $\geq 40$ for adults. We categorized the number of comorbidities as 0 vs. $\geq 1$ for children and $0,1,2$, $\geq 3$ for adults. Demographics, clinical variables and referrals were summarized with percentages, and chi-square tests were used to test for differences in referrals by demographic and clinical variables. Analyses were conducted with SAS Enterprise Guide 7.1. This study was approved by the Maine Medical Center IRB.

\section{RESULTS}

A total of 20,461 patients met inclusion criteria and were included in the analysis, including 2,780 pediatric patients $<18$, and 17,681 adult patients (18 or older). The mean age of the pediatric group was 11, and the mean BMI was 97.2 percentile for age. The mean age of the adult group was 52, and the mean BMI was 36 . The most common comorbidities documented among children were acanthosis nigricans (3.0\%), sleep apnea (1.9\%), and hypertension (1.4\%). The most common comorbidities documented among adults were hypertension (46.8\%), diabetes (18.9\%), and sleep apnea (14.3\%). See Table 1 (Pediatric patients with $\mathrm{BMI} \geq 95 \%$ for age) and Table 2 (Adult patients with $\mathrm{BMI} \geq 30$ ) for complete demographic characteristics of our study population.

Among all children with obesity, $31.2 \%$ had obesity documented on the problem list, and $12.5 \%$ received a referral to either a dietitian or obesity medicine specialist. Among all adults with obesity, $54.2 \%$ had obesity documented on the problem list, and $8.4 \%$ received a referral to a dietitian or an obesity medicine specialist (Figure 1). 


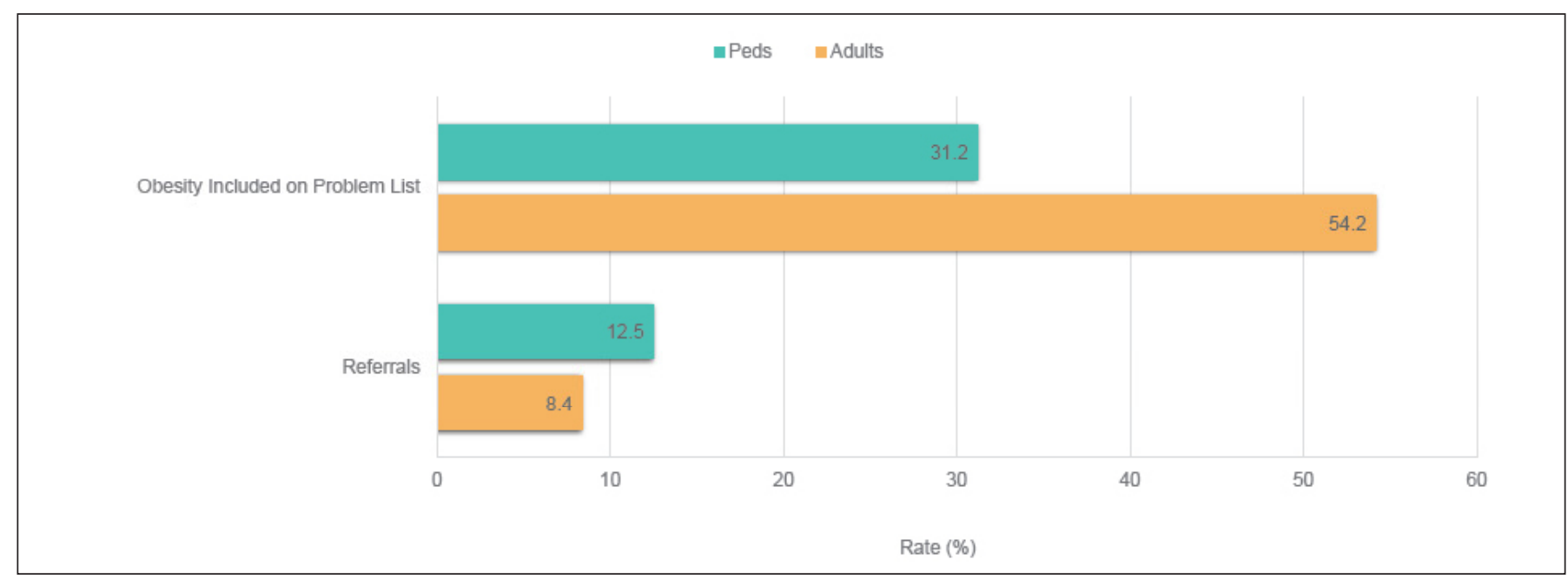

Figure 1. Documentation of Obesity on the Problem List and Referral Rates Among Children and Adults with Obesity

For both children and adults, obesity appearing on the problem list was significantly associated with higher percent of referral to an obesity medicine specialist or dietitian. Children with documented obesity on the problem list received referrals at a rate of $20.2 \%$ compared to only $9.0 \%$ in children without this clinical condition listed on the problem list $(p<$ 0.0001 ) (Figure 2). Adults with documented obesity received referrals at a rate of $12.1 \%$ compared to only $3.9 \%$ in adults without documentation on the problem list $(p<0.0001)$ (Figure 3$)$.

For children there was no significant difference in referral rates based on sex. For adults there was a significant difference in referral rates based on sex. Women were referred at a higher rate of $10.3 \%$ compared to men at $6.2 \%(p<0.0001)$ (Table 3$)$.

Significantly more referrals were made for both children and adults with a higher BMI. Children with a $\mathrm{BMI} \geq 99$ percentile received referrals at a rate of $26.6 \%$ compared to only $8.6 \%$ for children with a BMI between the 95th and 98th percentile ( $p<$ 0.0001 ) (Figure 2). Adults with a $\mathrm{BMI} \geq 40$ received referrals at a rate of $19.9 \%$ compared to only $5.8 \%$ for adults with a BMI $<40(p<0.0001)$ (Figure 3$)$.

Children and adults were also referred at higher rates if they had a greater number of comorbidities on the problem list. Children with $\geq 1$ comorbidity received referrals at a rate of $20.2 \%$ compared to $10.7 \%$ for children with 0 comorbidities $(p<0.0001)$
(Figure 2). Adults with $\geq 3$ comorbidities received referrals at a rate of $22.68 \%$ compared to $5.13 \%$ for adults with 0 comorbidities ( $p<0.0001)$ (Figure 3 ).

Each of the individual comorbidities for adults had a significant association with higher referral rates except for hyperglycemia and physical deconditioning. Adults with metabolic syndrome were referred at a rate of $37.8 \%$ compared to $8.1 \%$ for those without $(p<0.0001)$. Adults with acanthosis nigricans were referred at a rate of $25.6 \%$ compared to $8.3 \%$ for those without ( $p<$ 0.0001 ). Adults with PCOS were referred at a rate of $20.0 \%$ compared to $8.2 \%$ for those without ( $p<$ $0.0001)$. Adults with sleep apnea were referred at a rate of $18.5 \%$ compared to $6.7 \%$ for those without $(p<0.0001)$. Adults with diabetes were referred at a rate of $15.9 \%$ compared to $6.6 \%$ for those without ( $p$ $<0.0001$ ). Adults with hypertension were referred at a rate of $9.3 \%$ compared to $7.6 \%$ for those without $(p<0.0001)$ (Table 4).

\section{DISCUSSION}

Current USPSTF recommendations call on primary care providers to screen all pediatric (age 6 and older) and adult patients for obesity and offer or refer those that screen positively for intensive counseling and multicomponent behavioral intervention (grade $\mathrm{B}$ recommendation). Despite this, our study reveals that the overall documentation and referral rates for pediatric and adult patients remains low. 
Journal of Maine Medical Center, Vol. 3 [2021], Iss. 2, Art. 4

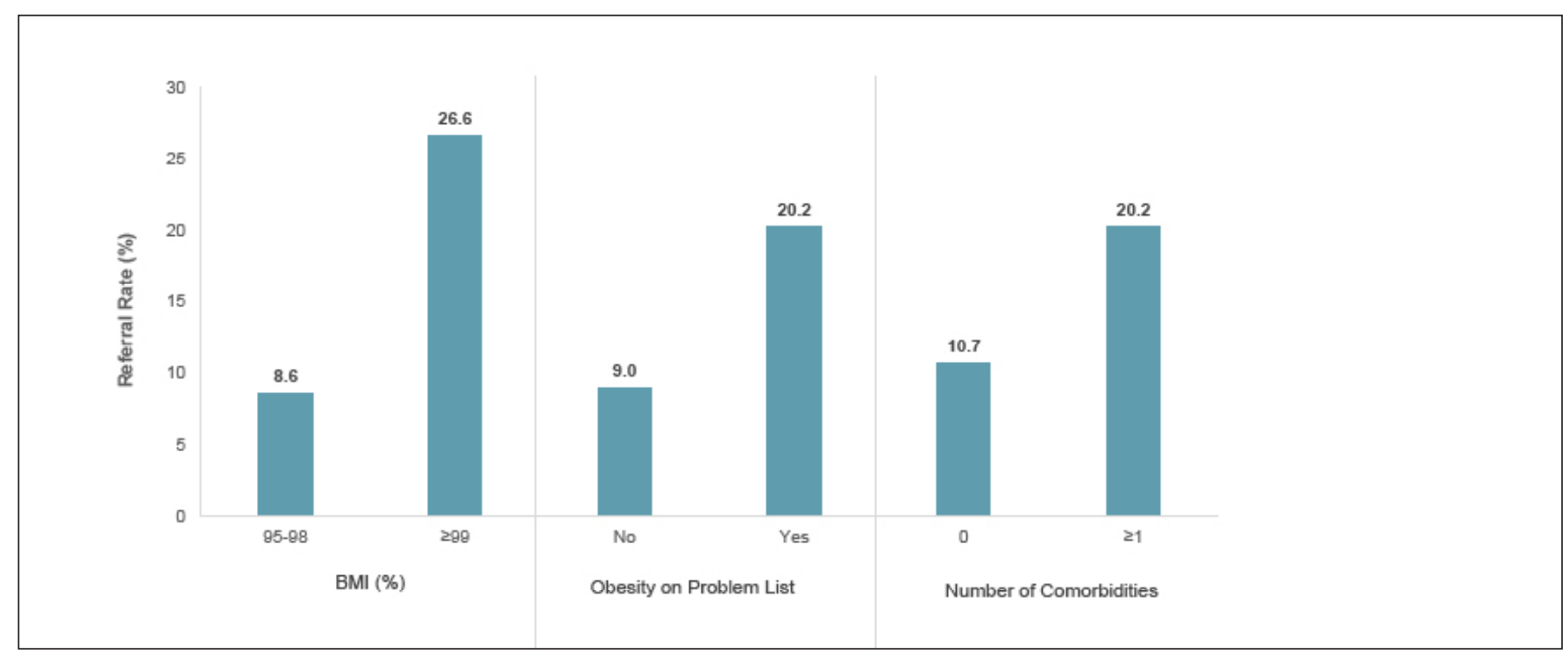

Figure 2. Pediatric Referral Rates by Characteristic

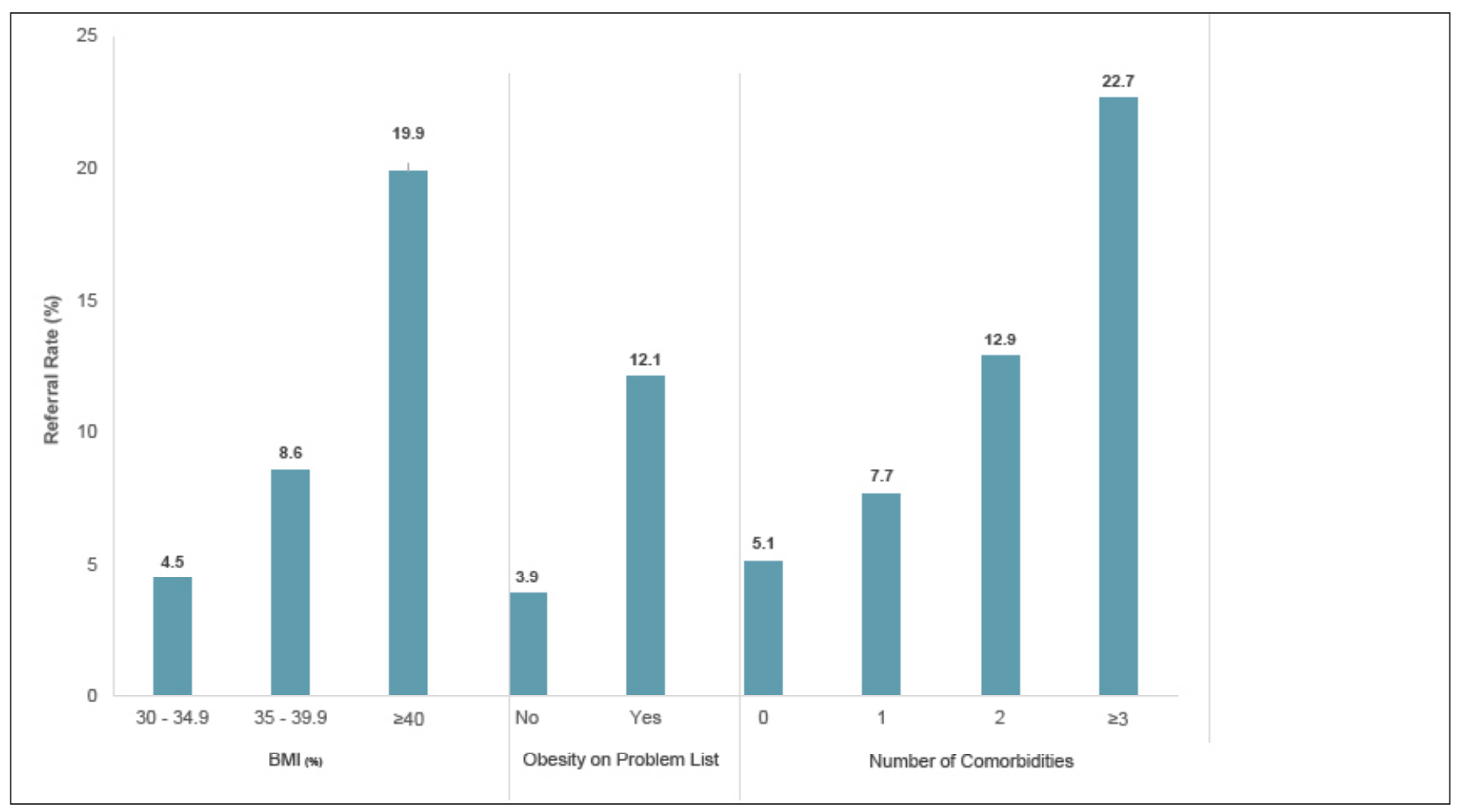

Figure 3. Adult Referral Rates by Characteristic 
Table 1. Pediatric Patients with High BMI $(\geq 95 \%)$ for Age $(\mathrm{N}=2780)$

\begin{tabular}{|c|c|c|}
\hline & No. & $\%$ \\
\hline \multicolumn{3}{|l|}{ Age, y } \\
\hline$<10$ & 944 & 34 \\
\hline $11-17$ & 1836 & 66 \\
\hline \multicolumn{3}{|l|}{ BMI percentile } \\
\hline $95-98$ & 2175 & 78.2 \\
\hline 99 & 605 & 21.8 \\
\hline \multicolumn{3}{|l|}{ Sex } \\
\hline Male & 1540 & 55.4 \\
\hline Female & 1240 & 44.6 \\
\hline Obesity on problem list (any) & 868 & 31.2 \\
\hline \multicolumn{3}{|l|}{ Type of obesity on problem list } \\
\hline Morbid obesity due to excess calories & 101 & 3.6 \\
\hline Other obesity due to excess calories & 100 & 3.6 \\
\hline Drug-induced obesity & 0 & 0 \\
\hline Morbid obesity with alveolar hypoventilation & 1 & 0 \\
\hline Overweight & 242 & 8.7 \\
\hline Other obesity & 0 & 0 \\
\hline Obesity, unspecified & 455 & 16.4 \\
\hline \multicolumn{3}{|l|}{ Comorbidities } \\
\hline Diabetes, type II & 10 & 0.4 \\
\hline Metabolic syndrome & 6 & 0.2 \\
\hline Polycystic ovarian syndrome & 21 & 0.8 \\
\hline Acanthosis nigricans & 83 & 3 \\
\hline Hyperglycemia & 5 & 0.2 \\
\hline Hypertension & 40 & 1.4 \\
\hline Obstructive sleep apnea & 52 & 1.9 \\
\hline Physical deconditioning & 1 & 0 \\
\hline Sedentary lifestyle & 0 & 0 \\
\hline Dietary counseling & 0 & 0 \\
\hline Exercise counseling & 0 & 0 \\
\hline \multicolumn{3}{|l|}{ Total comorbidities } \\
\hline 0 & 2591 & 93.2 \\
\hline 1 & 165 & 5.9 \\
\hline 2 & 20 & 0.7 \\
\hline$\geq 3$ & 4 & 0.2 \\
\hline Referral, any below & 347 & 12.5 \\
\hline Countdown Clinic & 283 & 10.2 \\
\hline Dietician & 344 & 12.4 \\
\hline Weight and Wellness & 4 & 0.1 \\
\hline
\end{tabular}

Abbreviations: BMI, body mass index; PCOS, polycystic ovarian syndrome. 
Table 2. Adult Patients with High BMI $(\geq 30)(N=17681)$

\begin{tabular}{|c|c|c|}
\hline & No. & $\%$ \\
\hline \multicolumn{3}{|l|}{ Age, y } \\
\hline $18-25$ & 1268 & 7.2 \\
\hline $26-40$ & 3570 & 20.2 \\
\hline $41-64$ & 8375 & 47.4 \\
\hline $65-79$ & 3817 & 21.6 \\
\hline $80+$ & 651 & 3.7 \\
\hline \multicolumn{3}{|l|}{ BMI category } \\
\hline $30-34.99$ & 9886 & 55.9 \\
\hline 35-39.99 & 4505 & 25.5 \\
\hline$\geq 40$ & 3290 & 18.6 \\
\hline \multicolumn{3}{|l|}{ Sex } \\
\hline Male & 8236 & 46.6 \\
\hline Female & 9445 & 53.4 \\
\hline Obesity on problem list (any) & 9635 & 54.5 \\
\hline \multicolumn{3}{|l|}{ Type of obesity on problem list } \\
\hline Morbid obesity due to excess calories & 1616 & 9.1 \\
\hline Other obesity due to excess calories & 531 & 3 \\
\hline Drug-induced obesity & 11 & 0.1 \\
\hline Morbid obesity with alveolar hypoventilation & 14 & 0.1 \\
\hline Overweight & 610 & 3.5 \\
\hline Other obesity & 107 & 0.6 \\
\hline Obesity, unspecified & 7131 & 40.3 \\
\hline \multicolumn{3}{|l|}{ Comorbidities } \\
\hline Diabetes, type II & 3334 & 18.9 \\
\hline Metabolic syndrome & 164 & 0.9 \\
\hline Polycystic ovarian syndrome & 335 & 1.9 \\
\hline Acanthosis nigricans & 43 & 0.2 \\
\hline Hyperglycemia & 596 & 3.4 \\
\hline Hypertension & 8270 & 46.8 \\
\hline Obstructive sleep apnea & 2533 & 14.3 \\
\hline Physical deconditioning & 146 & 0.8 \\
\hline Sedentary lifestyle & 0 & 0 \\
\hline Dietary counseling & 6 & 0 \\
\hline Exercise counseling & 2 & 0 \\
\hline \multicolumn{3}{|l|}{ Total comorbidities } \\
\hline 0 & 7557 & 42.7 \\
\hline 1 & 5887 & 33.3 \\
\hline 2 & 3258 & 18.4 \\
\hline 3 & 904 & 5.1 \\
\hline$\geq 4$ & 75 & 0.4 \\
\hline Referral, any below & 1482 & 8.4 \\
\hline Countdown Clinic & 4 & 0 \\
\hline Dietician & 988 & 5.6 \\
\hline Weight and Wellness & 686 & 3.9 \\
\hline
\end{tabular}

Abbreviations: BMI, body mass index; PCOS, polycystic ovarian syndrome. 
Table 3. Referral Rates by Sex

\begin{tabular}{lcc}
\hline & $\%$ & P value \\
\hline Pediatric referral rates & & \\
\hline Male & 11.8 & \\
\hline Female & 13.2 & 0.24 \\
\hline Adult referral rates & & \\
\hline Male & 6.2 & \\
\hline Female & 10.3 & $<.0001$ \\
\hline
\end{tabular}

Table 4. Adult Referral Rates by Comorbidity

\begin{tabular}{|c|c|c|c|c|}
\hline & Total & No. & $\%$ & $P$ value \\
\hline \multicolumn{5}{|c|}{ Diabetes } \\
\hline Yes & 3334 & 530 & 15.9 & \\
\hline No & 14347 & 952 & 6.6 & $<.0001$ \\
\hline \multicolumn{5}{|c|}{ Hypertension } \\
\hline Yes & 8270 & 769 & 9.3 & \\
\hline No & 9411 & 713 & 7.6 & $<.0001$ \\
\hline \multicolumn{5}{|c|}{ Metabolic syndrome } \\
\hline Yes & 164 & 62 & 37.8 & \\
\hline No & 17517 & 1420 & 8.1 & $<.0001$ \\
\hline \multicolumn{5}{|c|}{$\begin{array}{l}\text { Polycystic ovarian } \\
\text { syndrome }\end{array}$} \\
\hline Yes & 335 & 67 & 20 & \\
\hline No & 17346 & 1415 & 8.2 & $<.0001$ \\
\hline \multicolumn{5}{|c|}{ Acanthosis nigricans } \\
\hline Yes & 43 & 11 & 25.6 & \\
\hline No & 17638 & 1471 & 8.3 & $<.0001$ \\
\hline \multicolumn{5}{|c|}{ Hyperglycemia } \\
\hline Yes & 596 & 53 & 8.9 & \\
\hline No & 17085 & 1429 & 8.4 & 0.65 \\
\hline \multicolumn{5}{|c|}{$\begin{array}{l}\text { Obstructive sleep } \\
\text { apnea }\end{array}$} \\
\hline Yes & 2533 & 470 & 18.6 & \\
\hline No & 15148 & 1012 & 6.7 & $<.0001$ \\
\hline \multicolumn{5}{|c|}{ Physical deconditioning } \\
\hline Yes & 146 & 15 & 10.3 & \\
\hline No & 17535 & 1467 & 8.4 & 0.37 \\
\hline
\end{tabular}


In support of prior research that suggests documenting obesity on the problem list promotes provider action in addressing obesity, our study shows that among both pediatric and adult populations, referrals for interdisciplinary care of obesity were more likely if the diagnosis of obesity was on the problem list. Given this positive correlation, it is important to recognize that providers may face barriers in including obesity on the problem list: extra work for documentation, concern that patients with obesity will be offended or hurt by seeing obesity on their problem list, and lack of awareness that obesity is not due only to personal behavioral factors.

The fear of offending patients is not unwarranted, as there is a significant stigma associated with overweight and obesity. Unfortunately, healthcare settings can be a significant source of weight stigma, which undermines patient's opportunities to receive effective care. Studies show that providers rate thinner patients as more likable and are more likely to spend more time counseling those patients than their overweight or obese counterparts (9). Studies also show that patients who experience weight stigma are more likely to engage in unhealthy eating behaviors and less likely to participate in regular physical activity (9).

Obesity is documented on the problem list more frequently for adults with obesity than children $(54.2 \%$ vs. $31.2 \%)$. Perhaps there is more reluctance among providers to label children as obese even when they meet the definition per BMI criteria. While the overall referral rates to an Obesity Medicine Specialist or dietitian are very low, the referral rate for children is higher than for adults despite lower rates of inclusion of obesity on the problem list (12.5\% vs. $8.4 \%)$. This may be in part related to the requirements for a referral to the adult obesity medicine clinic (BMI > 40 or BMI > 35 with comorbidities), but would not be expected to impact referrals to dietitians (no specific BMI requirement, but dietitian referrals may require certain comorbidities for reimbursement from insurance). The higher referral rate for children could be related to the stigma of obesity being stronger for adults. While society may tend to believe the cause and solution for obesity resides within individual control, it may be easier to accept that children's behaviors are shaped by their environment and factors outside of their control. If understanding of obesity places less blame on the individual, this may lead to the receipt of more effective care in the management of obesity.

The significant differences in referral rates among adult men and adult women with obesity are interesting. Our data only captures whether a referral was made, but it does not capture whether one was verbally offered and subsequently declined or whether those patients attended the initial consult. Although there is not a clinical reason to refer women at higher rates, it is possible that women are more likely to accept a referral from their primary care provider regardless of whether they intend to follow through, and men may be more likely to decline the referral before it is made. It is also possible that there is underlying gender bias at work. Could providers refer women more often because of subconscious bias that it is less acceptable for women to carry extra weight? Future research could look at referral completion rates and the factors that may be associated with a higher likelihood of a patient presenting for their initial consult.

A higher number of comorbidities for both children and adults was associated with a higher referral rate. The comorbidities were obtained through data on the use of ICD-10 diagnosis codes at any time during the study period. It makes sense that patients suffering from comorbidities associated with obesity itself would be more likely to receive a referral. Since our healthcare system operates under a single EHR, the diagnosis codes could have been entered by the primary care provider or the obesity medicine specialist. The individual diagnosis codes with the highest referral rates were metabolic syndrome, acanthosis nigricans, and PCOS; the comorbidities that were most common: hypertension, diabetes, and sleep apnea had lower rates of referral. Further work could investigate whether these diagnosis codes appeared before or after the referral and which provider noted them the primary care provider or a specialist.

Although the low rates of documentation of obesity and low rates of referral raise concern that primary care providers may be missing opportunities to identify their patients affected by obesity and take action to manage it, we were not able to capture whether providers were engaging in nutrition or exercise counseling during the office visits. Behavioral interventions are an important part of the treatment of obesity. While counseling is part 
of the comprehensive treatment model offered with the obesity medicine specialists, we did not collect data on separate referrals to behavioral health for obesity management due to the difficulty in extracting those referrals from referrals to behavioral health for other reasons. Our data is not able to capture whether referrals to a dietitian or obesity medicine specialist were offered but declined by patients. The USPSTF recommendations are based on evidence of moderate benefit in weight loss with receipt of "intensive" behavioral interventions, defined as $>26$ contact hours over 2 to 12 months. These interventions rarely took place in a primary care setting. So, while the primary care provider's role in managing obesity is important, this suggests effective care may require a multidisciplinary team.

An important limitation of this study design is that we cannot measure change in BMI, change in behavior, or clinical outcomes related to obesity. We recognize the significant challenges that both children and their families and adults face in having limited access to effective, intensive treatment interventions for obesity.

A strength of this study is the large study population, lending the power to examine which covariates were significantly associated with referrals. Also, because we are a large primary care system covering most communities in the most populated southern Maine counties, we are able to generalize about the experience of our full patient populations including those that may be more rural and less easily served by specialty care.

There is certainly significant room for improvement in documentation of obesity on the problem list of our patients, and opportunities for future studies to explore barriers to referring patients to intensive treatment interventions. We are fortunate to have obesity medicine specialists who treat pediatric and adult patients within our healthcare system, and we recognize that not all primary care providers have access to this kind of specialty care for their patients with obesity. It is also notable that we share Epic as an electronic health record, and it would be interesting to explore the potential for how our system could leverage this to provide a more coordinated model of care between primary care providers and specialists. Care options for obesity include behavioral counseling, nutrition counseling, and obesity medicine specialist care - and current studies show effective interventions typically occur outside of the primary care office. Documentation by primary care providers of obesity on the problem list correlates with a higher referral rate, so it may be of value to encourage more consistent documentation through provider education, as well as identify and address barriers to documentation through future research. We have a large population of both children and adults affected by obesity who, according to our data, may not be receiving the intensive treatment and support services they need. This represents a missed opportunity and a call to action at both a provider and systems level.

\section{CONCLUSIONS}

In a large multispecialty health system retrospective study, we found that children and adults with obesity are more likely to be referred to a dietitian or obesity medicine specialist when any one of the following conditions are met: obesity is on the problem list, if they have a higher BMI, or when they have more medical comorbidities.

\section{Conflict of Interest: None}

\section{REFERENCES}

1. National Center for Health Statistics. Prevalence of obesity and severe obesity among adults: United States, 2017-2018. NCHS Data Brief, no 360. 2020

2. The State of Obesity: Better Policies for a Healthy America 2020. https://www.tfah.org/wp-content/uploads/2020/09/ TFAHObesityReport_20.pdf. Accessed February 2021.

3. Jay M., Gillespie C., Ark T., et al. Do internists, pediatricians, and psychiatrists feel competent in obesity care?: Using a needs assessment to drive curriculum design. Journal of General Internal Medicine. 2008;23(7):1066-1070.

4. Banerjee ES, Gambler A, Fogleman C. Adding obesity to the problem list increases the rate of providers addressing obesity. Family Medicine. 2013;45(9):629-33.

5. Moyer MA. Screening for and management of obesity in adults: U.S. Preventive Services Task Force recommendation statement. Ann Intern Med. 2012; 157(5): 373-378

6. https://www.uspreventiveservicestaskforce.org/uspstf/document/ RecommendationStatementFinal/obesity-in-children-andadolescents-screening. Accessed February 2021.

7. Blair, S. N. Physical inactivity: the biggest public health problem of the 21st century. British Journal of Sports Medicine. 2009;43(1): 1-2.

8. Cyr PR, Haskins AE, Holt C, Hanifi J. Weighty Problems: Predictors of Family Physicians Documenting Overweight and Obesity. Family Medicine. 2016;48(3):217-221.

9. Am J Public Health. 2010;100:1019-1028. doi:10. 2105/ AJPH.2009.159491 